\title{
Retraction Note to: Application of Scientific Approaches for Evaluation of Quality of Learning Objects in eQNet Project
}

\author{
Eugenijus Kurilovas ${ }^{1,2,3}$ and Silvija Serikoviene ${ }^{1}$ \\ ${ }^{1}$ Institute of Mathematics and Informatics, Akademijos str. 4, 08663 Vilnius, Lithuania \\ ${ }^{2}$ Vilnius Gediminas Technical University, Sauletekio al. 11, 10223 Vilnius, Lithuania \\ ${ }^{3}$ Centre of Information Technologies in Education, Suvalku str. 1, 03106 Vilnius, Lithuania \\ eugenijus.kurilovas@itc.smm.1t, silvija.serikoviene@gmail.com
}

M.D. Lytras et al. (Eds.): WSKS 2010, Part I, CCIS 111, pp. 437-443, 2010.

(c) Springer-Verlag Berlin Heidelberg 2010

DOI 10.1007/978-3-642-16318-0_76

The paper starting on page 437 of this volume has been retracted as it is a duplicate of the paper starting on page 329 of the same volume. 